\title{
越南统计数据库的八十年通过 SciMath 项目
}

\author{
Nguyen Thanh Thanh Huyen, Le Tam Tri, Dong Minh Huyen
}

2021 年 5 月 1 日

D0I: 10.31219/osf. io/7y86n

报告 “越南数学研究的 80 年发展一一从 SciMath 数据中关于数学家, 他们的作 品以及他们的合作者的的初步见解 “项目, 以及数据于 2020 年 11 月 13 日在 越南高级数学研究所呈现。

该报告总结了近 80 年 自 1947 年以来一一这一年被视为越南现代数学诞生的一 个里程碑, 标志着 Le Van Thiem 教授在国际期刊上发表的第一篇研究论文。在 撰写本文时, 数据收集了自 1947 年以来的 1566 位越南数学家和 1492 位外国数 学家的 8372 份出版物。

该项目于 2019 年在 Ngo Bao Chau 教授和 VIASM 的首席执行官 Le Minh Ha 博士 的主持下提出, 取材于 Ngo 教授与 Vuong Quan Hoang 博士 (Phenikaa 大学) 的 讨论。同年 8 月, 建立了 SciMath 数据; 12 月, 启动并测试了 http://SciMath. aisdl. com 的网站。

该项目将于 2020 年初正式开始。要纳入 SciMath, 该出版物必须满足以下两个 条件: 首先, 必须至少有一位越南 (共同) 作者。其次, 该出版物必须在以下 至少一项数据中列出: MathSciNet, zbMATH, Scopus 或Web of Science。

报告中有一些值得注意的结果:

1960 年以前的科学出版物数量很少, 只有 3 人撰写了约 9 篇文章, 除了 Le Van Thiem 教授以外，是 Dang Dinh Ang 教授 和 Nguyen Dinh Ngoc 教授。自 1970 年代以来, 出版物的数量迅速而稳定地增加。

毫无意外, 文章数量最多的 2 种期刊是越南杂志 ( 《Acta Mathematica Vietnamica》和《越南数学杂志》) 。排名前 10 位的其他期刊显示了越南的 关键行业-优化, 分析或代数。（有关详细信息, 请参见表 4-按行业分类的期 刊和作者数量)。与其他期刊相比, 有两种越南期刊压倒性的越南出版物, 分 别为 838 种和 695 种, 而排名第三的杂志只有 158 种。 
对于一流的杂志, 在前 10 种期刊中仅发表了 97 篇文章, 在前 5 种期刊中发表 了 27 篇文章。直到 2010 年 Ngo 教授获得 Fields 奖后, 质量的转变才真正发 生。同年, 通过了一项国家数学发展重点计划, 有助于数学家创造了更好的工 作环境。一个明显的结果是, 在过去的几年中, 数学家的数量（导致作品的数 量和质量）急剧增加。如果在 1990 年代至 2000 年代之间, 每年的新数学家人 数仅为 30 人左右, 最近这一数字已增至 70 人左右, 有些年甚至超过了 100 人。 这被认为越南数学的一个好兆头。

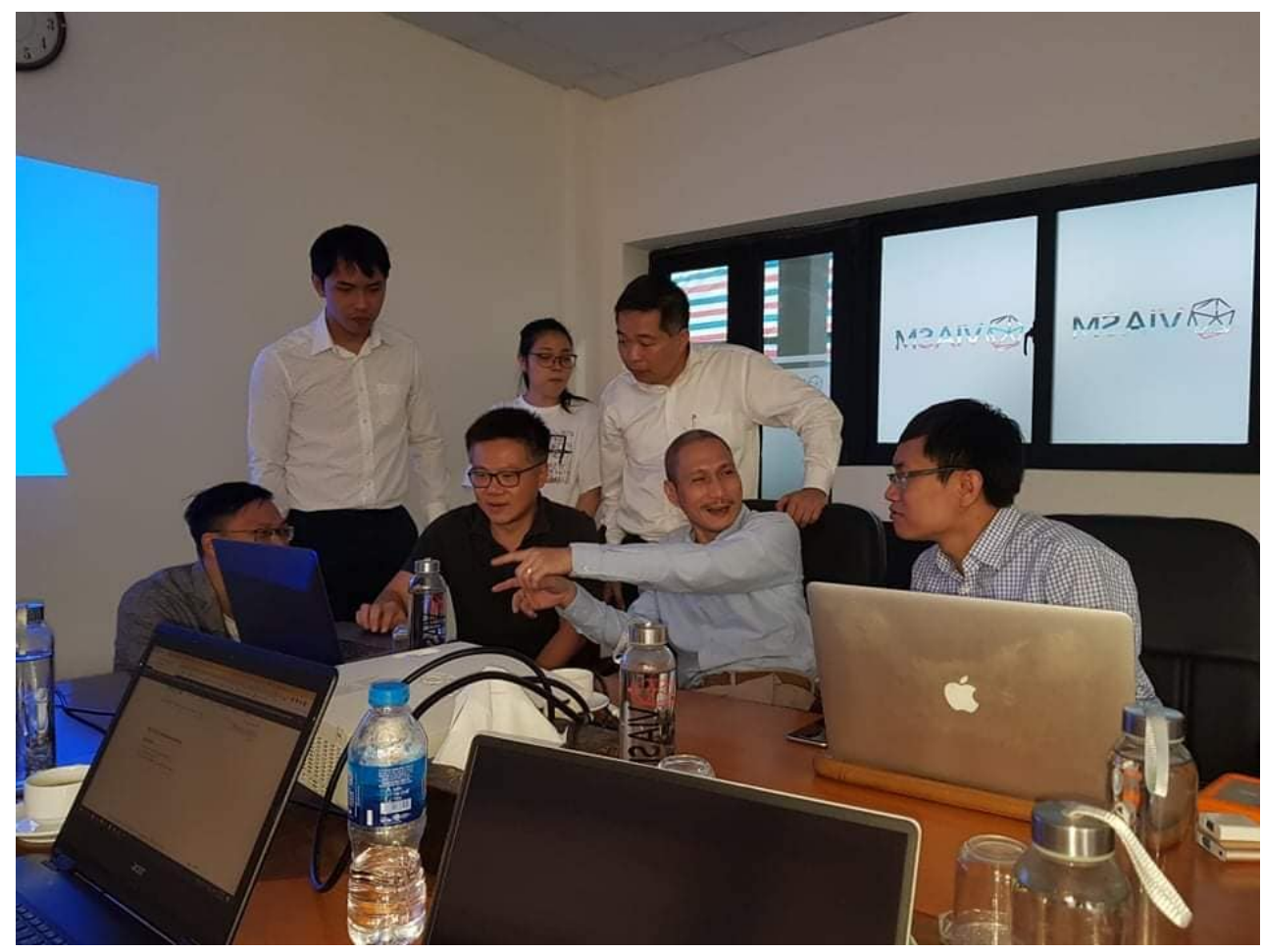

VIASM—-2020 2020 年 10 月 26 日

与研究和协作相关的一个显着点是, 我们的数学家喜欢独立工作或以小组形式 （2 个人， 3 个人）工作，并且小组之间彼此相关。SciMath 数据共有 3058 位 作者, 其中外国作者占了近一半, 这表明我国的研究发展严重依赖于外部。毫 不奇怪, 拥有悠久研究传统的数学学院—VAST 贡献了最多的出版物, 占已发 表著作总数的近三分之一。以下是河内科学大学, 河内教育大学和胡志明市自 然科学大学, 有 500 多种出版物。 
2020 年 12 月 22 日更新了一个好消息, 就是 2021-2030 年国家数学重点计划已 经通过了。作为一个从该计划中获得很多奖励的人, 我希望越南的数学在未来 的发展中将有许多突破和进一步发展。

\section{参考：}

Chau, N. B., Hoang, V. Q., Phuong, L. V., Hoa, L. T., Ha, L. M. , et al. (2020). The 80-year development of Vietnam mathematical research: Preliminary insights from the SciMath database on mathematicians, their works and their networks. arXiv preprint; arXiv:2011. 09328.

Huyen, N. T. T. (2020). ISR trình bày CSDL ngành toán tại Hội đồng Khoa học VIASM. Centre ISR, 18-11-2020. https://isr. phenikaauni. edu. vn/vi/post/tin-tuc/tin-tuc/sub-tin-tuc/isr-trinh-baycsdl-nganh-toan-tai-hoi-dong-khoa-hoc-viasm

Vuong, Q. H. (2018). The (ir)rational consideration of the cost of science in transition economies. Nature Human Behaviour, 2(1), 5.

Vuong, Q. H. (2019). Breaking barriers in publishing demands a proactive attitude. Nature Human Behaviour, 3(10), 1034.

Vuong, Q. H. (2020). Reform retractions to make them more transparent. Nature, $582(7811), 149$.

Vuong, Q. H., et al. (2018). An open database of productivity in Vietnam's social sciences and humanities for public use. Scientific Data, 5(1), 1180188. 